\title{
\begin{tabular}{l|l} 
Mibraries & DSpace@MIT
\end{tabular}
}

\author{
MIT Open Access Articles
}

\section{Measurement of the T+T Neutron Spectrum Using the National Ignition Facility}

The MIT Faculty has made this article openly available. Please share how this access benefits you. Your story matters.

Citation: Sayre, D. B., C. R. Brune, J. A. Caggiano, V. Y. Glebov, R. Hatarik, A. D. Bacher, D. L. Bleuel, et al. "Measurement of the T+T Neutron Spectrum Using the National Ignition Facility." Physical Review Letters 111, no. 5 (August 2013). (c) 2013 American Physical Society

As Published: http://dx.doi.org/10.1103/PhysRevLett.111.052501

Publisher: American Physical Society

Persistent URL: http://hdl.handle.net/1721.1/81399

Version: Final published version: final published article, as it appeared in a journal, conference proceedings, or other formally published context

Terms of Use: Article is made available in accordance with the publisher's policy and may be subject to US copyright law. Please refer to the publisher's site for terms of use. 


\title{
Measurement of the $T+T$ Neutron Spectrum Using the National Ignition Facility
}

\author{
D. B. Sayre, ${ }^{1, *}$ C. R. Brune, ${ }^{2}$ J. A. Caggiano, ${ }^{1}$ V. Y. Glebov,${ }^{3}$ R. Hatarik, ${ }^{1}$ A. D. Bacher, ${ }^{4}$ D. L. Bleuel, ${ }^{1}$ D. T. Casey, ${ }^{1}$ \\ C. J. Cerjan, ${ }^{1}$ M. J. Eckart, ${ }^{1}$ R. J. Fortner, ${ }^{1}$ J. A. Frenje, ${ }^{5}$ S. Friedrich, ${ }^{1}$ M. Gatu-Johnson, ${ }^{5}$ G. P. Grim, ${ }^{6}$ C. Hagmann, ${ }^{1}$ \\ J. P. Knauer, ${ }^{3}$ J. L. Kline, ${ }^{6}$ D. P. McNabb, ${ }^{1}$ J. M. McNaney, ${ }^{1}$ J. M. Mintz, ${ }^{1}$ M. J. Moran, ${ }^{1}$ A. Nikroo, ${ }^{7}$ T. Phillips, ${ }^{1}$ J. E. Pino, ${ }^{1}$ \\ B. A. Remington, ${ }^{1}$ D. P. Rowley, ${ }^{1}$ D. H. Schneider, ${ }^{1}$ V. A. Smalyuk, ${ }^{1}$ W. Stoeffl, ${ }^{1}$ R. E. Tipton, ${ }^{1}$ \\ S. V. Weber, ${ }^{1}$ and C. B. Yeamans ${ }^{1}$ \\ ${ }^{1}$ Lawrence Livermore National Laboratory, Livermore, California 94550, USA \\ ${ }^{2}$ Ohio University, Athens, Ohio 45701, USA \\ ${ }^{3}$ University of Rochester, Rochester, New York 14623, USA \\ ${ }^{4}$ Indiana University, Bloomington, Indiana 47405, USA \\ ${ }^{5}$ Massachusetts Institute of Technology, Cambridge, Massachusetts 02139, USA \\ ${ }^{6}$ Los Alamos National Laboratory, Los Alamos, New Mexico 87545, USA \\ ${ }^{7}$ General Atomics, San Diego, California 92121, USA
}

(Received 25 May 2013; published 2 August 2013)

\begin{abstract}
Neutron time-of-flight spectra from inertial confinement fusion experiments with tritium-filled targets have been measured at the National Ignition Facility. These spectra represent a significant improvement in energy resolution and statistics over previous measurements, and afford the first definitive observation of a peak resulting from sequential decay through the ground state of ${ }^{5} \mathrm{He}$ at low reaction energies $E_{\text {c.m. }} \lesssim$ $100 \mathrm{keV}$. To describe the spectrum, we have developed an $R$-matrix model that accounts for interferences from fermion symmetry and intermediate states, and show these effects to be non-negligible. We also find the spectrum can be described by sequential decay through $\ell=1$ states in ${ }^{5} \mathrm{He}$, which differs from previous interpretations.
\end{abstract}

DOI: 10.1103/PhysRevLett.111.052501

PACS numbers: 25.55. $-\mathrm{e}, 24.10 .-\mathrm{i}, 52.57 .-\mathrm{z}$

Investigations of the neutron spectrum from the low energy $T(t, 2 n) \alpha$ reaction [1] have spanned six decades, driven by interests ranging from fusion research [4-6] to the neutron-neutron scattering length [7]. The spectrum provides information about interactions among final-state particles, while the $T(t, 2 n) \alpha$ yield extracted from spectral measurements assists efforts to understand thermonuclear environments created in the laboratory. Reaction yields test the modeling of inertial confinement fusion, for example, in experiments designed to measure mixing between the ablator and fuel [8,9]. Also, since the particle spectrum results from three-body decay its sensitivity to different breakup mechanisms has long been of interest [10]. Energy resolution, backgrounds, and statistics have presented major experimental challenges in the past, and a modern shortage of tritium beams has required developing alternative approaches to study the spectrum. Still, large uncertainties remain in the spectrum, and disparate accounts of its underlying breakup processes exist $[5-7,10]$.

The particle spectrum from the $T(t, 2 n) \alpha$ reaction has been described previously by statistical and/or sequential processes [5-7,10-14]. Statistical decays uniformly populate the available phase space, producing a neutron spectrum in the shape of an ellipse. Sequential decays proceed through intermediate states formed by $n-\alpha\left({ }^{5} \mathrm{He}\right)$ or $n-n$ (correlated dineutron) interactions. (Note: the distinction between statistical and sequential mechanisms can become ambiguous, since broad intermediate states can also fill phase space uniformly.) When transitions are made through ${ }^{5} \mathrm{He}$, the neutrons emitted first are spread over the decay width of the intermediate state by energymomentum conservation. The distribution of neutrons from ${ }^{5} \mathrm{He}$ is further broadened by recoil, with a shape reflecting the angular correlation between neutrons. For correlated dineutron decays, the phase-space distribution of energies is modified by an $s$-wave singlet interaction between final-state neutrons.

Discussions in the literature of sequential decay through ${ }^{5} \mathrm{He}$ must include the interference effects arising from a final state with identical fermions. These order-ofemission effects have been well established with studies involving the $3 \alpha$ breakup of ${ }^{12} \mathrm{C}$ [15-17] and also have been applied to the sequential proton decay of ${ }^{6} \mathrm{Be}[18]$. The coherence between accessible states in ${ }^{5} \mathrm{He}$ should be considered as well. For the ground $J^{\pi}=3 / 2^{-}$and firstexcited $1 / 2^{-}$states in ${ }^{5} \mathrm{He}$, this produces an interference in the angular correlation between neutrons and, by extension, the spectrum. We [19] have formulated sequential decay processes within the $R$-matrix framework $[20,21]$, where both interferences can be incorporated. This model reveals substantial changes occurring from antisymmetrization.

Experimental studies of the neutron spectrum have taken a variety of approaches to investigate the $T(t, 2 n) \alpha$ reaction, covering a range in center-of-mass energies from $E_{\text {c.m. }}=750 \mathrm{keV}$ [11] down to nearly zero energy $[4,5]$. 
In addition to earlier accelerator work [7,10,11,14], fusion experiments based on muon catalyzation $[4,5]$ and inertial confinement [6] have been conducted. Although the precise details of detectors differed among experiments, most have deduced the neutron spectrum from either proton or deuteron recoils $[4-6,10,11]$. It is interesting to note that only three groups have reported their experimental energy resolution (full width at half maximum): 0.5 [14], 1.3 [6], and $2.8 \mathrm{MeV}$ [11]. The work presented in this Letter has combined the inertial confinement approach with time-offlight technique to perform measurements resolved to $280 \mathrm{keV}$ [22] - a factor of $\approx 2$ improvement over any previous experiment.

The first reported measurement of the neutron spectrum $\left(E_{\text {c.m. }}=110 \mathrm{keV}\right)[10]$ was interpreted as a statistical distribution, accompanied by a much smaller $(\approx 10 \%)$ ${ }^{5} \mathrm{He}$ ground-state contribution. Wong et al. [14] later determined at slightly higher energies $(250 \mathrm{keV})$ that $70 \%$ of decays proceeded through correlated dineutron emission, with the remainder split between the ground $(20 \%)$ and first-excited $(10 \%)$ states of ${ }^{5} \mathrm{He}$. As stipulated in their paper the branching ratios were directly tied to an assumption of a strong dineutron channel. The predominance of that channel is contrary to the findings of Poutissou et al. [7] $(20 \mathrm{keV})$, who stated that any $n-n$ correlations were masked by those from $n$ - $\alpha$, and Matsuzaki et al. [5] $(\approx 0 \mathrm{keV})$, who emphasized the importance of $n$ - $\alpha$ interactions. Yet the conclusions of both groups were strongly model dependent as no definitive signature of $n$ - $\alpha$ interactions was visible in their data, such as the peak at 8.7 MeV in the spectra of Refs. [10,14] from sequential decays through the ground state of ${ }^{5} \mathrm{He}$. The significance of these interactions at low reaction energies was further brought into question by a recent Letter [6] that reported no observable $n$ - $\alpha$ effects at $E_{\text {c.m. }}=23 \mathrm{keV}$. Casey et al. [6] pointed out the diminishing strength of the ${ }^{5} \mathrm{He}$ groundstate peak with reaction energy, from Wong et al. to Allen et al. [10] to Poutissou et al., who also reported no appreciable contribution from this channel. In addition, the correlated dineutron distribution of Refs. [13,14] was found to describe the measurements of Casey et al. Utilizing a new experimental capability, we report, in this Letter, the first direct observation of the peak from the ${ }^{5} \mathrm{He}$ ground-state channel at low energies. Further, with the $R$-matrix model, we find the main features in these new measurements can be described by sequential decay through $\ell=1$ states in ${ }^{5} \mathrm{He}$.

The energy distribution of particles emitted by reactions in unbound states can be described with $R$-matrix methods, as given by Barker [21]. But when a reaction leads to a final state containing identical particles, the standard formalism must be adapted to treat an amplitude with exchange symmetry. For the low energy $T(t, 2 n) \alpha$ reaction, coupling tritons in an $s$ wave to form a $0^{+}$state, the amplitude can be generalized as

$$
\begin{aligned}
\mathcal{M}_{\nu_{1} \nu_{2}}^{\ell J}= & \sum_{m_{\ell} m_{\ell}^{\prime} m} \frac{(-1)^{J+m}}{\sqrt{2 J+1}}\left(\ell m_{\ell} \frac{1}{2} \nu_{1} \mid J m\right) \\
& \times\left(\ell m_{\ell}^{\prime} \frac{1}{2} \nu_{2} \mid J-m\right) Y_{\ell m_{\ell}}\left(\hat{p}_{1}\right) Y_{\ell m_{\ell}^{\prime}}\left(\hat{p}_{23}\right) \\
& \times \frac{e^{-i\left(\Phi_{1}+\Phi_{23}\right)} \sqrt{\frac{P_{1} P_{23}}{p_{1} p_{23}}} \sum_{\lambda} \frac{A_{J \lambda} \gamma_{J \lambda}}{E_{J \lambda}-E_{23}}}{1-\left(B_{J}-S_{23}+i P_{23}\right) R_{J \lambda}}
\end{aligned}
$$

The numerical subscripts in Eq. (1) reflect the decay process: emission of neutron 1 and the subsequent disintegration of ${ }^{5} \mathrm{He}$ to neutron 2 and $\alpha$ particle 3 . For a given $J^{\pi}$ in ${ }^{5} \mathrm{He}$ and orientations $\nu$ of emitted neutrons, summations are performed over possible angular momentum quantum numbers. Clebsch-Gordan coefficients along with spherical harmonics $Y_{\ell m_{\ell}}$, which depend on projections of momenta $p$ in the three-body $\hat{p}_{1}$ and recoil center-of-mass $\hat{p}_{23}$ systems, describe the angular correlation between neutrons. Only the ground $3 / 2^{-}$and first-excited $1 / 2^{-}$states of ${ }^{5} \mathrm{He}$ are directly accessible from reaction energies relevant here, and for both cases $\ell=1$ neutrons are ejected in each stage of the breakup. Energy levels $E_{J \lambda}$, reducedwidth amplitudes $\gamma_{J \lambda}$, boundary values $B_{J}$, and feeding factors $A_{J \lambda}$ are the $R$-matrix parameters contained above, and all except the $A_{J \lambda}$ factors can be constrained with high precision $n-\alpha$ scattering data [23]. The remaining terms are calculable for a given channel radius and partial wave: the hard sphere phase shift $\Phi$ and the penetration $P$ and shift $S$ factors.

Exchanging neutron indices in Eq. (1) yields a second expression, which is necessary to construct the antisymmetric amplitude. The energy distribution is obtained by summing the amplitude over $J$, squaring the magnitude, then summing over $\nu_{1}$ and $\nu_{2}$, and multiplying by the three-body phase-space factor [24]. Interferences from symmetry and intermediate states originate from cross terms in the squared amplitude. Lastly, to describe kinematically incomplete measurements, such as the spectra considered here, an integration is performed over angles of the unobserved neutron. Figure 1 presents spectra that result from the procedure outlined above. The spectrum calculated for the $1 / 2^{-}$partial wave (middle panel) illustrates the importance of antisymmetry, which leads to a $\approx 30 \%$ contribution.

Inertial confinement experiments were conducted at the National Ignition Facility. Here, temporally shaped laser beams were used to generate a soft $\mathrm{x}$-ray field that ablatively imploded a spherical plastic capsule filled with $99.9 \%$ tritium gas ( $0.1 \%$ deuterium). Using time-of-flight detectors, an ion temperature of $k_{B} T=3.3(3) \mathrm{keV}$ [or an effective energy for the $T(t, 2 n) \alpha$ reaction of $E_{\text {c.m. }}=$ 16(1) $\mathrm{keV}$ ] within the burn volume was extracted from thermal broadening of nearly monoenergetic $14 \mathrm{MeV}$ neutrons produced by the $T(d, n) \alpha$ reaction. A total neutron yield in excess of $10^{13}$ was produced over the $\approx 200 \mathrm{ps}$ 


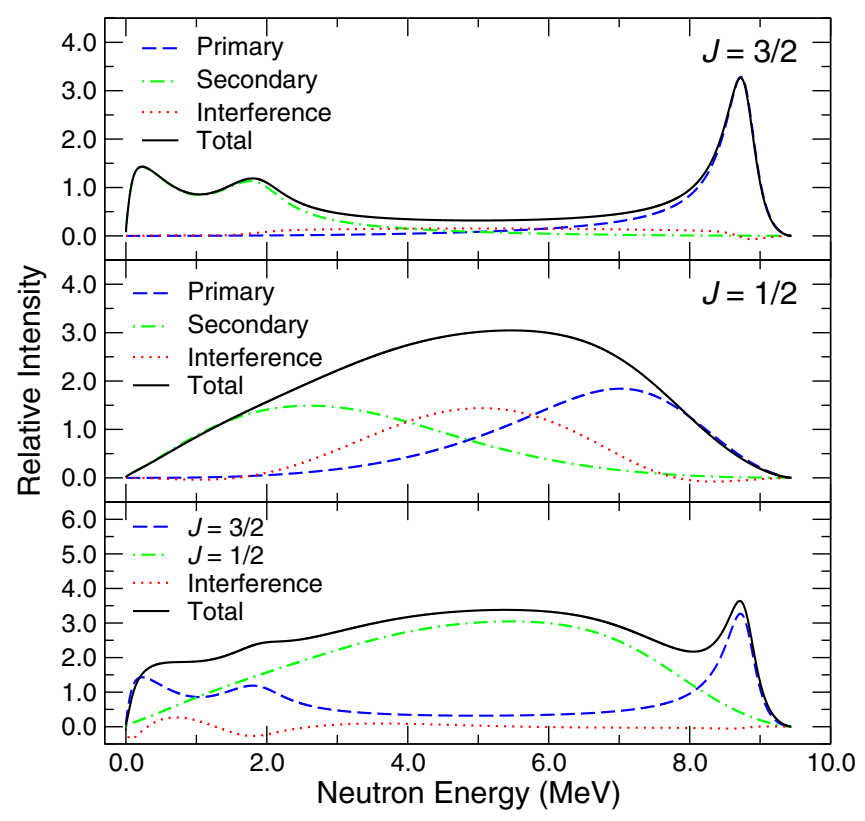

FIG. 1 (color online). $\quad R$-matrix spectra resulting from the fitted feeding factors are shown above. Decays through partial waves of the ground and first-excited states of ${ }^{5} \mathrm{He}$ are shown in the top and middle panels, respectively. Each partial wave contains an interference term from antisymmetrization, which is proportional to the overlap between primary and secondary distributions. For the $J=1 / 2^{-}$partial wave, the interference term makes $a \approx 30 \%$ contribution. The bottom panel displays the coherent sum of both partial waves. An interference created within the neutron angular correlation manifests itself in the spectrum. The feeding factors determine the sign of this interference.

duration of thermonuclear reactions, affording excellent statistics and timing. Measurements have also been performed with a capsule containing a deuterium-to-tritium ratio of $3: 1$. These data serve to establish the neutroninduced background from residual deuterium in the capsules, and show a relatively flat baseline several decades below the preceding $T(d, n) \alpha$ peak (see Fig. 2, bottom panel).

Neutron spectra were recorded by two $10 \mathrm{~cm}$ by $5 \mathrm{~cm}$ xylene scintillators, positioned over $20 \mathrm{~m}$ from the target chamber center along separate lines of sight [25]. Each line was doubly collimated with $1 \mathrm{~m}$ of steel: the first collimator defined a $8.3 \mathrm{~cm}$ diameter opening on the detector face and the second was designed to clean up after the first. A $1.3 \mathrm{~cm}$ thick attenuator $(95 \% \mathrm{~W}, 3 \% \mathrm{Ni}$, and $2 \% \mathrm{Fe})$ covered each line of sight to suppress the photon background created during implosions. Attenuation and scattering of the neutron spectrum from intervening material has been taken into account with MCNP [26] simulations, and corrections were $\lesssim 10 \%$ above $2 \mathrm{MeV}$.

Implosions are singular events which generate tremendous neutron fluxes, therefore, the standard methods of

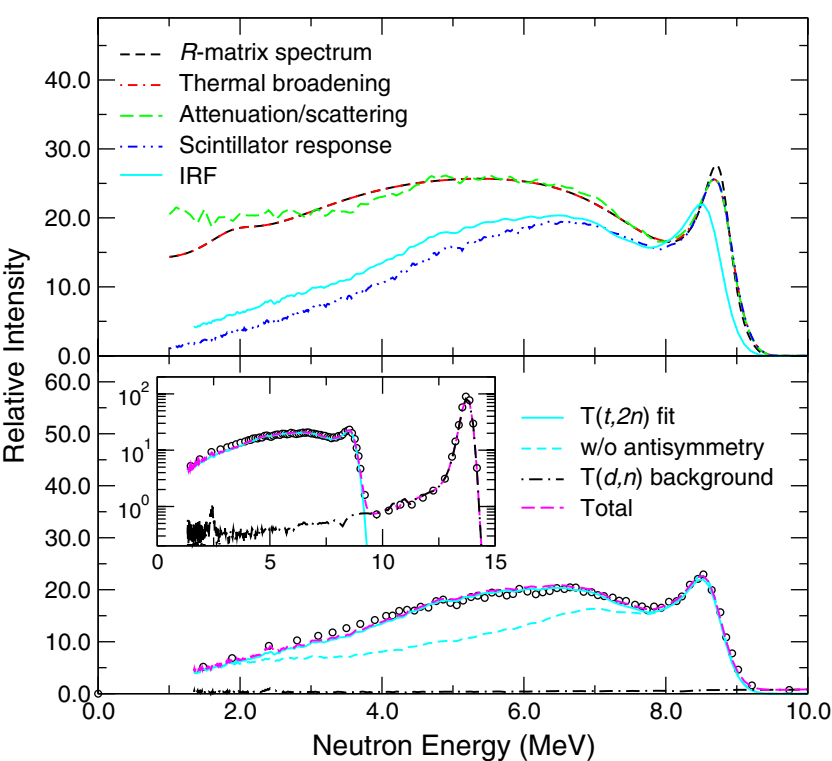

FIG. 2 (color online). Experimental corrections applied to the modeled spectrum, as discussed in the text, are displayed in successive order (top panel). The combined fit is plotted over energy-binned data from the $22 \mathrm{~m}$ detector (bottom panel), in the region of interest and over the full range of neutron energies. Statistical errors are approximately the size of the data points. Transitions through the ground state of ${ }^{5} \mathrm{He}$ produce a peak at $\approx 8.7 \mathrm{MeV}$; the broad distribution peaked around $6 \mathrm{MeV}$ can largely be described by the first-excited state in ${ }^{5} \mathrm{He}$. For reference, the short-dashed (cyan) curve shows the effect of not including antisymmetry. The background from deuterium contamination, which is illustrated better in the inset, has a minimal effect in the region of interest.

pulse counting are not applicable. Detectors were operated in current mode and their signals were digitized with $1 \mathrm{GHz}$ oscilloscopes. Interpreting current mode data requires knowledge of the impulse response functions (IRFs) from detectors. As the light decay time of the scintillator is rather insensitive to particle type, short pulses (100 ps) of $\mathrm{x}$ rays have been used to measure the IRFs. Further refinements have been made to account for neutron transport in the scintillator. Constructed IRFs have been validated over many measurements of the $T(d, n) \alpha$ peak, in which good fits to data $\left(\chi_{\nu}^{2} \approx 1\right)$ were obtained with the expected distribution from thermal broadening [27]. Careful attention has also been given to describing light production in the scintillator over observed neutron energies. Consistency checks of the scintillator response have been performed in situ with activation foils, which agree with the scintillators to $8 \%$.

The $R$-matrix spectrum was fitted simultaneously to data from both detectors. This method combines neutron statistics and averages over systematic differences between measurements. The fitting algorithm first thermally broadens a trial spectrum then adjusts for attenuation or scattering and scintillation response. Next, for each detector, the 
algorithm transforms the modified spectrum from energy into time of flight and convolves with the IRFs. The top panel of Fig. 2 illustrates these experimental corrections. A $\chi^{2}$ minimization of the resulting spectrum to data determines free parameters with the MINUIT2 package [28]. Four feeding factors $A_{J \lambda}$ were allowed to vary in the fit; all remaining parameters were fixed to values reported by Stammbach and Walter [23].

Data corresponding to neutron energies $\lesssim 1 \mathrm{MeV}$ were excluded from the analysis presented in Fig. 2 because of limited detector sensitivity. Digitizer noise from oscilloscopes represented the main source of statistical uncertainty in the data. Fluctuations due to neutron events were much smaller, as $\approx 10^{6}$ neutrons interacted with the scintillator to produce the signal in the region of interest. Systematic uncertainties have been investigated for ion temperature, attenuation or scattering, region of interest, and scintillator response. These uncertainties become more important for data below 4.5 MeV.

Separate analyses were undertaken for each systematic error at its $1 \sigma$ deviation, and the sum of these individual errors $(16 \%)$ was added in quadrature with uncertainties determined from the fitting routine $(1 \%)$ to give a total error of $16 \%$. The best fit results in a $\chi^{2}=1440$ for 660 data points and 4 free parameters $\left(\chi_{\nu}^{2}=2.2\right)$, and comparable values of $\chi_{\nu}^{2}$ result for each detector. This fit determines branching ratios between the $3 / 2^{-}$and $1 / 2^{-}$states of $29 \pm 16 \%$ and $71 \mp 16 \%$, respectively. The error in the ratio is anticorrelated to maintain unity. Note that the ratio is largely insensitive to the interference between partial waves, as can be seen in the bottom panel of Fig. 1. While differences between the fitted curve and data appear in several locations, the model describes the main features in the spectrum quite well, particularly in the region of the spectrum that is least susceptible to systematic errors $\geq 4.5 \mathrm{MeV}$.

New spectra from inertial confinement fusion experiments with enriched tritium targets have been measured at the National Ignition Facility. These measurements provide conclusive evidence for $n$ - $\alpha$ interactions in the $T+T$ neutron spectrum produced by thermonuclear environments, through the first direct observation of a neutron peak from transitions via the ground state of ${ }^{5} \mathrm{He}$. A description of the data was obtained with an $R$-matrix model that accounts for the coherence between intermediate states and fermion symmetry, which was shown in this Letter to be non-negligible. Fits of the model to the spectrum show a large contribution from the $1 / 2^{-}$partial wave in ${ }^{5} \mathrm{He}$, which differs from the dominant role found for either correlated dineutron emission [6] or statistical decay [10]. Future work will investigate the possible role of dineutron and/or sequential $s$-wave emission within the framework of the new $R$-matrix approach.

Although a reasonable description of the data was obtained with the $R$-matrix model, we acknowledge that the present analysis has limitations as it represents a two-body approximation to what is strictly a three-body problem. Also, the interpretation of the neutron singles spectrum can be subject to ambiguities that only kinematically complete measurements distinguish. We hope these new data encourage further work in theory and experiment, and improve evaluations of fusion experiments.

We thank the operation teams at the National Ignition Facility and Lawrence Livermore National Laboratory Tritium Facility for enabling the current investigation. This work was performed under the auspices of Lawrence Livermore National Security, LLC, under Contract No. DE-AC52-07NA27344.

*sayre4@1lnl.gov

[1] We consider the low energy $T(t, 2 n) \alpha$ reaction over a region $E_{\text {c.m. }} \lesssim 100 \mathrm{keV}$ where the $s$-wave channel is dominant and the astrophysical $S$ factor is flat [2,3].

[2] R.E. Jarmie and R.E. Brown, Nucl. Instrum. Methods Phys. Res., Sect. B 10-11, 405 (1985).

[3] C. Angulo and P. Descouvemont, Nucl. Phys. A639, 733 (1998).

[4] W.H. Breunlich et al., Muon Catal. Fusion 1, 121 (1987).

[5] T. Matsuzaki et al., Phys. Lett. B 557, 176 (2003).

[6] D. T. Casey et al., Phys. Rev. Lett. 109, 025003 (2012).

[7] R. Larose-Poutissou and H. Jeremie, Nucl. Phys. A218, 559 (1974).

[8] D. C. Wilson, P. S. Ebey, T. C. Sangster, W. T. Shmayda, V. Yu. Glebov, and R. A. Lerche, Phys. Plasmas 18, 112707 (2011).

[9] V. A. Smalyuk (unpublished).

[10] K. W. Allen, E. Almqvist, J. Dewan, T. Pepper, and J. Sanders, Phys. Rev. 82, 262 (1951).

[11] S. J. Bame and W. T. Leland, Phys. Rev. 106, 1257 (1957).

[12] N. Jarmie and R.C. Allen, Phys. Rev. 111, 1121 (1958).

[13] B. Lacina, J. Ingley, and D. W. Dorn, Lawrence Radiation Lab, University of California, Livermore Report No. 7769, 1965.

[14] C. Wong, J. D. Anderson, and J. W. McClure, Nucl. Phys. 71, 106 (1965).

[15] P. Swan, Rev. Mod. Phys. 37, 336 (1965).

[16] D. P. Balamuth, R. W. Zurmühle, and S. L. Tabor, Phys. Rev. C 10, 975 (1974).

[17] H. O. U. Fynbo et al., Phys. Rev. Lett. 91, 082502 (2003).

[18] D. F. Geesaman, R. McGrath, P. Lesser, P. Urone, and B. VerWest, Phys. Rev. C 15, 1835 (1977).

[19] C. R. Brune (unpublished).

[20] A. Lane and R. Thomas, Rev. Mod. Phys. 30, 257 (1958).

[21] F. C. Barker, Aust. J. Phys. 41, 743 (1988); we replace $G_{\lambda b c}^{1 / 2}$ by $P^{1 / 2} A_{\lambda}$ in his Eq. (27).

[22] The intrinsic detector resolution for $8.7 \mathrm{MeV}$ neutrons $(180 \mathrm{keV})$ is thermally broadening by $220 \mathrm{keV}$, resulting in an effective resolution of $280 \mathrm{keV}$. 
[23] T. Stammbach and R. L. Walter, Nucl. Phys. A180, 225 (1972); we fix our $R$-matrix parameters for $E_{J \lambda}, \gamma_{J \lambda}, B_{J}$, and channel radius to the values listed in their Table 2.

[24] G. G. Ohlsen, Nucl. Instrum. Methods 37, 240 (1965); the three-body phase space term is given by his Eq. (40).

[25] J. A. Caggiano (unpublished).
[26] R. Forster et al., Nucl. Instrum. Methods Phys. Res., Sect. B 213, 82 (2004).

[27] L. Ballabio, J. Kallne, and G. Gorini, Nucl. Fusion 38, 1723 (1998).

[28] F. James and M. Winkler, Minuit2: Function Minimization and Error Analysis (CERN, Geneva 2004). 